\title{
COMP-JT WITH DYNAMIC CELL SELECTION, GLOBAL PRECODING MATRIX AND IRC RECEIVER FOR LTE-A
}

\author{
Heba Raafat Ahmed ${ }^{1}$, Essam Sourour ${ }^{2}$ and Hassan M. Elkamchouchi ${ }^{3}$ \\ ${ }^{1}$ Department of Electrical Engineering, Pharos University, Alexandria, Egypt \\ ${ }^{2,3}$ Department of Electrical Engineering, Alexandria University, Alexandria, Egypt
}

\begin{abstract}
Coordinated multi-point transmission and reception (CoMP) is introduced in LTE-A to mitigate co-channel interference and improve the cell-edge user experience. In this paper we propose a joint transmission scheme for LTE-COMP and we enhance the performance of CoMP with two techniques: 1-dynamic MIMO cell selection and 2-closed loop MIMO with global precoding matrix selection. A cell-edge user selects the base stations that jointly transmit the desired signal from the available ones (we assumed 3). The user also selects the closed loop precoding matrices for MIMO in a joint fashion to fit the independent MIMO channels from two base stations (eNBs). In addition, edge users are likely to be subject to severe Cochannel interference from eNBs outside the joint transmission set.To address co-channel interference from the base station(s) that are not included in CoMP joint transmission set, the user equipment employs Minimum Mean Squared Error receiver with Interference Rejection Combining (MMSE-IRC). We illustrate the effect of fading correlation between elements of the transmit and receive antennas. Also, the effect of the desired to interference eNB power ratio in case of medium correlation for 3 and 4 layers using MMSE$I R C$ receiver is studied. Also we compare the BER performance for 3 and 4 layers in case of different values of the desired to interference eNB power ratio. Simulation results show that the performance of CoMP with cell selection considerably improves the performance. Also, global selection of the precoding matrices outperforms local selection. In addition, using MMSE-IRC gives much better performance than the conventional minimum mean square error (MMSE) in the presence of co-channel interference.
\end{abstract}

\section{KEYWORDS}

LTE-A; CoMP; MIMO; Joint transmission; Codebook; Capacity; MMSE; MMSE-IRC.

\section{INTRODUCTION}

The Long Term Evolution (LTE) Release 10 (Rel-10) broadband network is developed by the Third Generation Partnership Project (3GPP) and denoted as LTE-Advanced (LTE-A). LTE-A is expected to be the dominating mobile communication system in the near future. LTE-A targets 1 $\mathrm{Gb} / \mathrm{s}$ downlink (DL) and $500 \mathrm{Mb} / \mathrm{s}$ uplink (UL) throughputs to meet the IMT-Advanced requirements [1]-[5].

The performance improvements of LTE-Advanced are achieved with advanced physical layer techniques including carrier aggregation, enhanced. Interference coordination techniques, and enhanced multiple-antenna schemes (MIMO) [6]-[9].

In 3GPP LTE/LTE-A Release 8-10, partial DL channel state information is provided to the eNB transmitter through the closed loop MIMO mode. Codebook-based MIMO precoding technique

DOI : 10.5121/ijwmn.2015.7303 
has been used to reduce the feedback overhead [6]. The user equipment (UE) selects and feeds back the precoding matrix index (PMI) together with channel quality indicator (CQI) to the eNB. In this paper we extend the closed-loop MIMO concept to be used with CoMP-JT. [3].

So there are two ways for mitigating interference and improving the cell-edge user performance in Long Term Evolution-Advanced (LTE-A) system. First method is coordinated multi-point transmission and reception (CoMP) and second method is interference rejection combining (IRC) receiver, which is explained below.

One of the major enhancements in LTE-A is the employment of CoMP technology. In the downlink, CoMP allows multiple eNBs to transmit to a UE collaboratively. This is done through: a) Dynamic Point Selection (DPS), i.e., dynamic selection of the transmission point, b) Joint Transmission (JT), i.e., transmitting the same information from multiple eNBs to the same UE coherently, or c) Coordinated Scheduling/Beam-forming (CS/CB) [10],[11]. eNBs communicate with one another through the backhaul network such as X2 interface via fiber optics. Figures 1, 2, and 3 show three different schemes for CoMP [12]. Among these three types of DL CoMP techniques this paper is interested in JT. JT is particularly promising in the presence of cochannel interference because the signals transmitted from multiple eNBs are coherently combined by the UE in a constructive manner, achieving high SINR and throughput for the UE $[13,14]$.

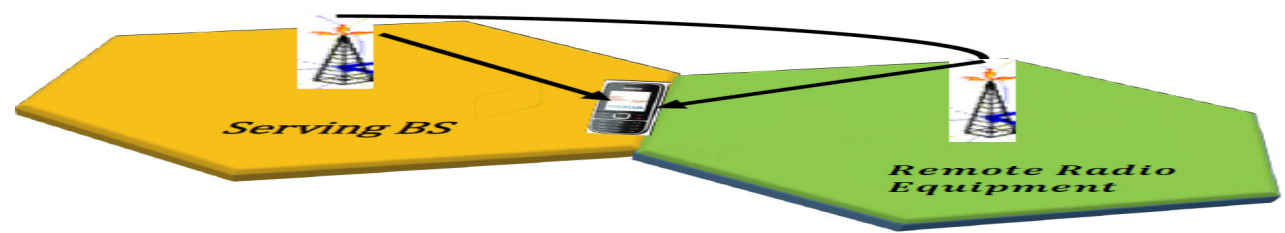

Figure 1. Joint Transmission cooperative (JT).

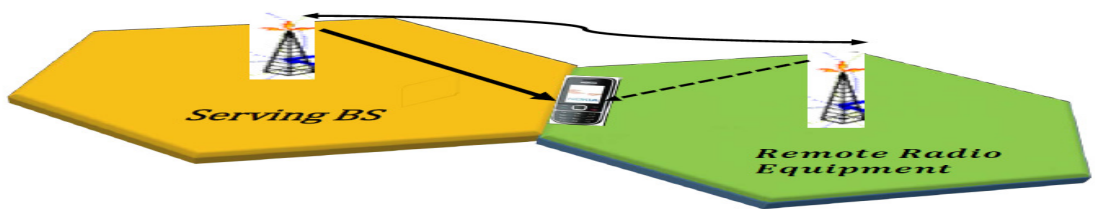

Figure 2. Dynamic Point Selection cooperative (DPS).

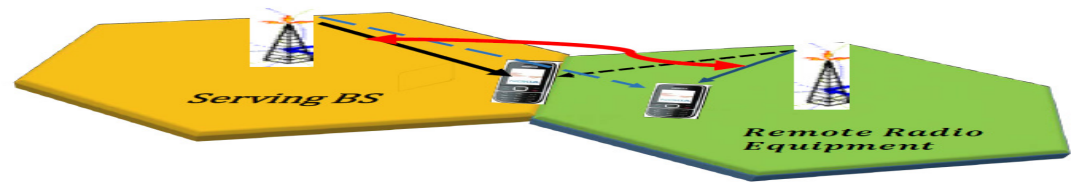

Figure 3. Coordinated scheduling/beam forming (CS/CB).

In our previous work, e.g., [15], we studied the performance of downlink CoMP-JT with closedloop MIMO in LTE-A networks. Edge UEs receive the desired signal from two eNBs: serving eNB and remote eNB. Each eNB applies a precoding matrix to the vector of transmitted modulation symbols. For compatibility with LTE specifications, the precoding codebook of LTEA [16] is used. We denote the conventional method for selecting the precoding matrix as the local 
precoding scheme. In this scheme the UE selects the precoding matrix for each eNB based on its corresponding channel, as depicted in figure 4 . On the other hand, in the global precoding scheme the UE selects the two precoding matrices for the serving and remote eNBs jointly to fit the distributed channel from the two eNBs. Both the local and global precoding require the same number of feedback bits. However, the global precoding scheme requires more search in the UE. To enhance CoMP performance, in this paper we consider the case when the UE is allowed to select the two jointly transmitting eNBs which makes considerable improvement. We show that combining the cell selection and precoding matrix selection techniques alleviate the severity of the interferenceThe cost is extra UE processing, extra feedback bits and backhaul overhead.

To further combat inter-cell interference the UE employs Interference Rejection Combining (IRC) based on the Minimum Mean Square Error (MMSE) criteria. IRC has been recently proposed to mitigate co-channel interference for edge users [17]-[21]. The conventional MMSE receiver treats the interference as being independent across the receive antennas (i.e., white interference). MMSE-IRC is a straightforward extension to the MMSE receiver employing the correlation matrix of the interfering signal across the receive antennas. Due to its simplicity, MMSE-IRC has replaced MMSE as the baseline MIMO receiver in LTE-A systems [22].

In this paper we couple the CoMP, which is the interference mitigation method from the network side, with MMSE-IRC as the interference mitigation method from the UE side.

To confirm the achieved performance gain we also study the effect of fading correlation among MIMO antennas. In addition, the effect of the desired to interference eNB power ratio in case of medium correlation for 3 and 4 layers using MMSE-IRC receiver is studied. Also we compare the BER performance for 3 and 4 layers in case of different values of the desired to interference eNB power ratio.

\subsection{Related Work}

It is known that, at low mobility, closed-loop MIMO outperforms open-loop MIMO (obviously at the cost of feedback overhead) [6]. Enhancing CoMP-JT performance with closed loop MIMO is generally suggested in the literature $[13,14]$. However, specific implementation, performance analysis and tradeoffs have not been sufficiently studied. In [11] closed loop MIMO precoding is considered for CoMP. A global precoding matrix codebook is created using a clustering algorithm from a large number of channel samples, generated through simulation. While the designed codebook matches well to the considered channel model it is not a standard LTE-A. In [23] Adaptive and distributed CoMP scheduling algorithm, in conjunction with open-loop MIMO, is proposed which could operate in either JT or CS/CB modes. In order to maximize the sum-rate of UEs under JT mode, beam-forming matrix is calculated using maximum capacity criteria. In [12] a general formulation of CoMP JT and DPS is provided. Edge users can switch between the modes of CoMP or fall back to the single eNB based on channel condition. However, neither one of the above mentioned references (or, to the best knowledge of the authors, any other CoMP reference) studied the LTE-A codebook in a closed loop MIMO scheme with CoMP. Moreover, most CoMP literature focuses on the capacity improvements without providing link level BER performance. Our current paper covers this gap.

The rest of this paper is organized as follows. Section II presents the system model employed in this paper. In section III we describe the CoMP-JT eNBs selection algorithm. In Section IV we present the algorithm for local and global precoding matrix selection with CoMP-JT. In Section V we provide the MMSE-IRC receiver structure. Section VI is devoted for the simulations result. Section VII concludes the paper. 


\section{COMP SYSTEM MODEL}

We consider the scenario where a cell-edge UE is located at equal distance from 3 eNBs. This UE, equipped with $n_{r}$ receive antennas, receives the desired signal from two eNBs: serving eNB and remote eNB. The third eNB becomes a source of inter-cell interference. Each eNB is equipped with $n_{t}$ transmit antennas and applies a precoding matrix to the vector of transmitted modulation symbols with $n_{l}$ layers. For LTE compatibility, the precoding codebook of LTE-A [16] is used. The received signal at subcarrier $k$ at the UE is given by:

$$
\mathbf{y}(k)=\left(\mathbf{H}_{s, k} \mathbf{W}_{s}+\mathbf{H}_{r, k} \mathbf{W}_{r}\right) \mathbf{s}(k)+\mathbf{H}_{i, k} \mathbf{W}_{i} \mathbf{x}(k)+\mathbf{v}(k)
$$

Where $\mathbf{s}(k)$ is a column vector of size $n_{l}$ of desired symbols at subcarrier $k$. The covariance matrix of $\mathbf{s}(k)$ is given by $\mathbf{C}_{s}=\mathbf{I}_{n_{l}}$, where $\mathbf{I}_{n_{l}}$ is the identity matrix of size $n_{l}$. Similar to $\mathbf{s}(k), \mathbf{x}(k)$ is a vector of interfering symbols with the same size and covariance matrix. Also, in (1) $\mathbf{H}$ is the channel matrix with size $n_{r} \mathrm{X} n_{t}$ and $\mathbf{W}$ is the precoding matrix with size $n_{t} \mathrm{X} n_{l}$. The subscripts $s, r$ and $i$ in $\left(\mathbf{H}_{s, k}, \mathbf{W}_{s}\right),\left(\mathbf{H}_{r, k}, \mathbf{W}_{r}\right)$ and $\left(\mathbf{H}_{i, k}, \mathbf{W}_{i}\right)$ indicate the serving, remote and interfering eNBs, respectively. The same precoding matrix is used for all the UE allocated subcarriers. Finally, $\mathbf{v}(k)$ is a vector with size $n_{r}$ representing the AWGN with covariance matrix $\mathbf{C}_{v}=\gamma^{-1} \mathbf{I}_{n_{r}}$, where $\gamma$ is the average SNR in each receive antenna due to each desired eNB. To simplify notation, in what follows we may drop the subcarrier index $k$. The 3 channel matrices $\mathbf{H}$ in (1) are independent and identically distributed (i.i.d). Each channel matrix is correlated according to $\mathbf{H}=\mathbf{R}_{U E}^{1 / 2} \mathbf{H}_{o} \mathbf{R}_{e N B}^{1 / 2}$, where $\mathbf{R}_{U E}$ and $\mathbf{R}_{e N B}$ are the UE and eNB antenna correlation matrices, respectively. Each element in the $n_{r} \times n_{t}$ channel matrix $\mathbf{H}_{o}$ is independent complex Gaussian with zero mean and unit variance.

\section{DYNAMIC CELL SELECTION}

We consider the scenario where the UE is allowed to select the serving and remote eNBs in (1) from the 3 near-by eNBs. This requires overhead in the backhaul network to make the UE data available to any 2 eNBs. In addition, at least in our implementation, the UE should acquire the channel state information for the 3 eNBs. We show in the numerical results that this additional complexity generously pays-back with performance enhancement.

Inspired by [24, 25], in this paper we propose to use capacity selection criteria for selecting the two serving eNBs. It is consistently shown ([24] and many similar papers) that other selection criteria (for example SINR) provide insignificant performance benefit. Denote the estimated channel matrices from the 3 eNBs to the UE as $\mathbf{H}_{e N B 1}, \mathbf{H}_{e N B 2}$ and $\mathbf{H}_{e N B 3}$ (including antenna correlation). Channel estimation is typically performed using the cell-specific reference signals transmitted from each eNB [26]. Define [X Y ] as the matrix formed by concatenating 2 matrices

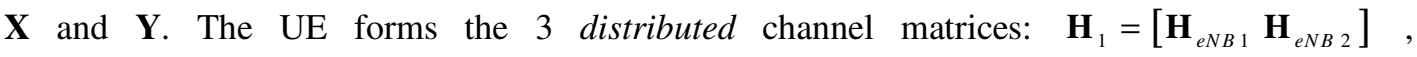
$\mathbf{H}_{2}=\left[\begin{array}{ll}\mathbf{H}_{e N B 1} & \mathbf{H}_{e N B_{3}}\end{array}\right]$ and $\mathbf{H}_{3}=\left[\begin{array}{ll}\mathbf{H}_{e N B} 2 & \mathbf{H}_{e N B_{3}}\end{array}\right]$, each with size $n_{r} \times 2 n_{t}$. The UE selects the two eNBs that maximize the distributed channel capacity. Specifically, the two jointly transmitting eNBs are selected as follows. 


$$
\begin{gathered}
C\left(\mathbf{H}_{m}\right)=\frac{1}{K} \sum_{k} \log \left(\operatorname{det}\left(\mathbf{I}_{2 n_{t}}+\frac{\gamma}{n_{t}} \mathbf{H}_{m}^{H} \mathbf{H}_{m}\right)\right), \\
\left.\mathbf{H}_{\text {selected }}=\underset{m \in\{1,2,3\}}{\arg \max }\left(\underset{\mathbf{H}_{m}}{C}\right)\right) .
\end{gathered}
$$

In (2), $K$ is the number of allocated subcarriers to the UE. Also $\mathbf{A}^{H}$ is the Hermitian of matrix $\mathbf{A}$. Note that (2) is not the accurate capacity formula since it does not take into account the interference caused by the third eNB (see for example equation (2) in [27]). However, with accurate formula no significant difference was found, while large matrix inversion was incurred. Once, the two jointly transmitting eNBs are decided the next step is to select their precoding matrices.

\section{CoMP-JT PRECODING MATRIX SELECTION}

We denote the conventional method for selecting the precoding matrix as the local precoding scheme. This is the scheme used in closed-loop MIMO without CoMP-JT. In this scheme the UE selects the precoding matrix for each eNB based on its corresponding channel. On the other hand, in the global precoding scheme the UE selects the two precoding matrices for the serving and remote eNBs jointly to fit the distributed channel from the two eNBs. Both the local and global precoding schemes require the same number of feedback bits. However, the global precoding scheme requires more processing in the UE. For example, if the number of transmit antennas in each eNB is 4, the number of precoding matrices in LTE-A codebook is 16 [16]. If the number of receive antennas in the UE is 4 , then in the local precoding scheme the UE searches the two codebooks separately for each eNB based on each corresponding $4 \mathrm{X} 4$ channel. On the other hand, in the global precoding scheme the UE searches a global codebook comprising of $16 \times 16=256$ precoding matrices of all possible combinations of the two local codebooks. In this case the distributed channel is $4 \times 8$ joint channel.

\subsection{CoMP-JT With Local Precoding Matrix}

The received signal from the two eNBs is given by (1). The UE separately selects the best $\mathbf{W}_{s}$ based on $\mathbf{H}_{s}$ and the best $\mathbf{W}_{r}$ based on $\mathbf{H}_{r}$ according to their respective channel capacity. Several metrics have been proposed in the literature [24, 25]. SINR maximization criteria provided the best performance it most cases. Capacity maximization is very slightly inferior but less complex. Hence, in this paper we employ the capacity maximization criteria. The maximum capacity criterion is given by:

$$
\begin{gathered}
C\left(\mathbf{W}_{m}\right)=\frac{1}{K} \sum_{k} \log \left(\operatorname{det}\left(\mathbf{I}_{n_{l}}+\gamma \mathbf{W}_{m}^{H} \mathbf{H}_{k}^{H} \mathbf{H}_{k} \mathbf{W}_{m}\right)\right), \\
\mathbf{W}_{\text {select }}=\arg \max _{W_{m} \in\{W\}}\left(C\left(\mathbf{W}_{m}\right)\right) .
\end{gathered}
$$

The selections in (4) and (5) are repeated separately for $\mathbf{H}_{k}=\mathbf{H}_{s, k}$ and $\mathbf{H}_{k}=\mathbf{H}_{r, k}$ to get $\mathbf{W}_{s}$ and $\mathbf{W}_{r}$, respectively. The set of precoding matrices to search is that of LTE-A [16]. 


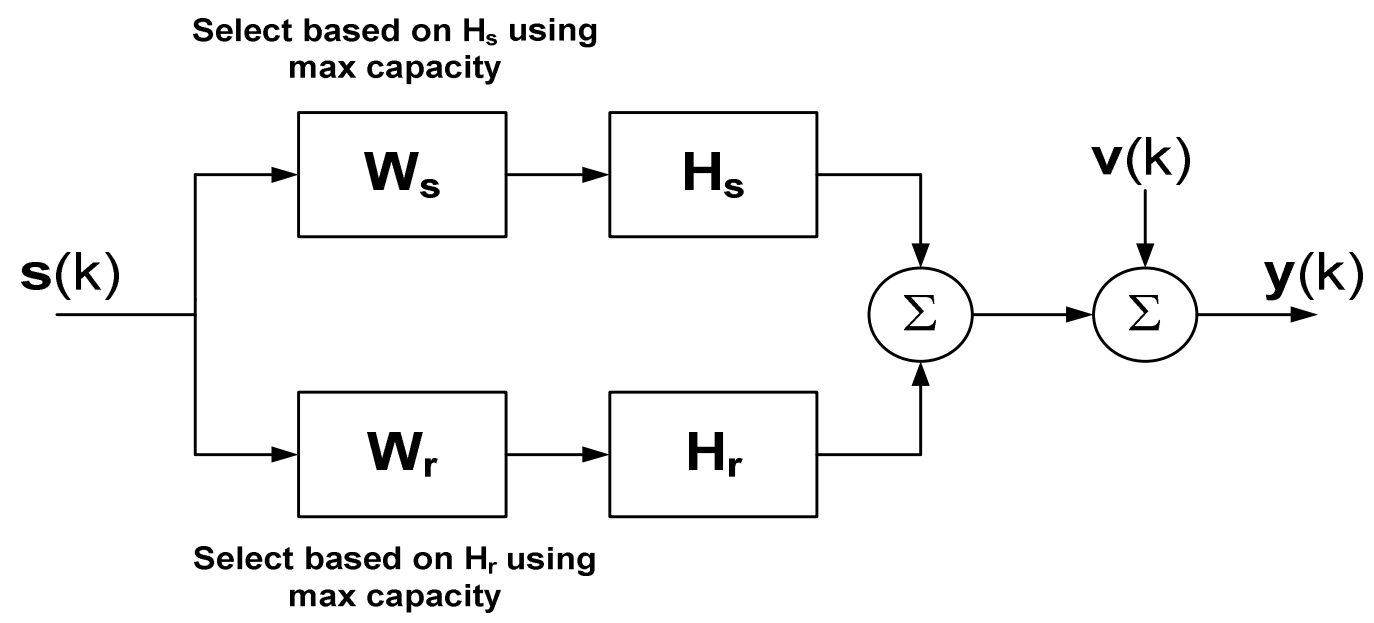

Figure 4. CoMP-JT system with locally selected precoding matrices

\subsection{CoMP-JT With Global Precoding Matrix}

An alternative approach is to consider the two eNBs as a large distributed antenna array, and the two channels as one distributed channel $\mathbf{H}$ with dimension $n_{r} \times 2 n_{t}$. Similar to [11] the UE should select a single global precoding matrix for this composite channel. We can rewrite (1) as:

$$
\mathbf{y}(k)=\mathbf{H}_{k} \mathbf{W} \mathbf{s}(k)+\mathbf{H}_{i, k} \mathbf{W}_{i} \mathbf{x}(k)+\mathbf{v}(k)
$$

In (6) $\mathbf{H}_{k}$ is the global channel at subcarrier $k$ and $\mathbf{W}$ is the global precoding matrix. These are given by:

$$
\mathbf{H}_{k}=\left[\mathbf{H}_{s, k} \mathbf{H}_{r, k}\right] \text { and } \mathbf{W}=\left[\mathbf{W}_{s}^{T} \mathbf{W}_{r}^{T}\right]^{T}
$$

In (7) $\mathbf{A}^{T}$ is the transpose of the matrix A. Now, the global precoding matrix $\mathbf{W}$ is selected using (4) and (5). However, the codebook to select from includes all possible combinations of $\mathbf{W}_{s}$ and $\mathbf{W}_{r}$. For example, with 4 transmit antennas the LTE-A codebook includes 16 precoding matrices. Hence, the global codebook includes $16 \times 16=256$ precoding matrices with all permutations.

\section{INTERFERENCE REJECTION COMBINING RECEIVER}

Recently, the problem of interference cancellation and suppression has attracted a lot of attention. Co-channel interference is mitigated by means of several techniques operating either at the network side (like CoMP) or at the UE receiver (like MMSE-IRC).

In Release 8 LTE the MMSE was considered the baseline MIMO receiver upon which the minimum performance requirements for the UE are set. This is based on the assumption that cochannel interference from adjacent cells is uncorrelated across the receive antennas. To mitigate the effect of co-channel interference release 11 LTE introduced MMSE-IRC receivers as the baseline. Fig.5 describe the scenarios where the MMSE receiver and MMSE-IRC receiver should be used. 


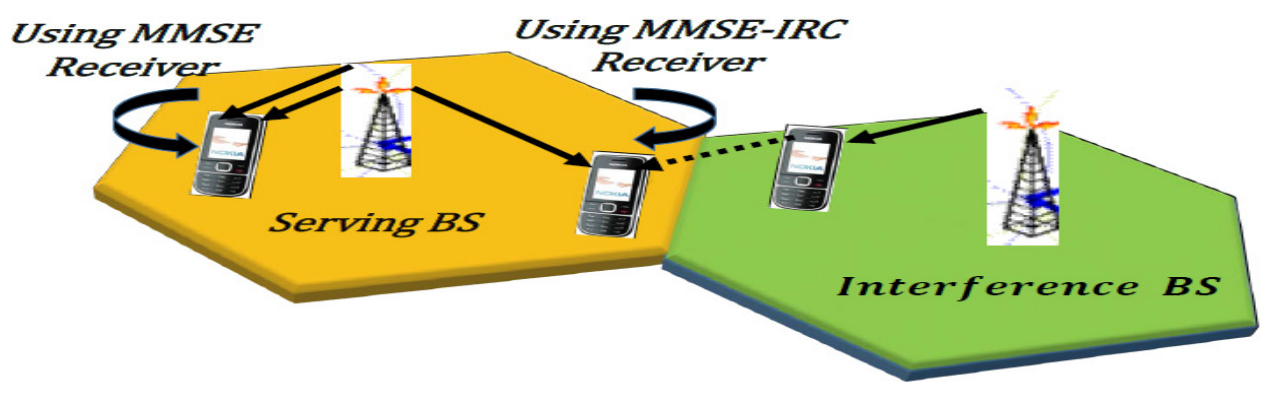

Figure 5. MMSE-IRC receiver.

Here, we employ MMSE-IRC with CoMP-JT. At this point the jointly transmitting eNBs and their precoding matrices have been selected. We now modify the received signal (1) as:

$$
\mathbf{y}(k)=\mathbf{G}(k) \mathbf{s}(k)+\frac{1}{\sqrt{f}} \mathbf{G}_{i}(k) \mathbf{x}(k)+\mathbf{v}(k) .
$$

Comparing to (1), $\mathbf{G}(k)=\mathbf{H}_{s, k} \mathbf{W}_{s}+\mathbf{H}_{r, k} \mathbf{W}_{r}$ is the composite channel matrix of the desired modulation symbols vector $\mathbf{s}(k)$ and $\mathbf{G}_{i}(k)=\mathbf{H}_{i, k} \mathbf{W}_{i}$ is the composite interference channel matrix of the interference modulation symbols vector $\mathbf{x}(k)$. The interference precoding matrix $\mathbf{W}_{i}$ is randomly selected from the LTE-A codebook [16].

A conventional method to reduce co-channel interference is power control. In (8) the added factor $f$ is the power ratio between the desired signal from each desired eNB and the interference signal. This can be considered a form of coordinated scheduling CoMP where the interfering eNB allocates the same subcarriers to a center UE and reduces the transmit power on these subcarriers. Note that in LTE-A power control is only for the data carrying subcarriers and not for the cellspecific reference signals. Hence, the process of joint transmission eNBs selection and precoding matrix selection described in sections III and IV above is not affected by power control.

The MMSE-IRC symbol estimator is given by [21]:

$$
\hat{\mathbf{s}}=\left(\mathbf{C}_{s}^{-1}+\mathbf{G}^{H} \mathbf{C}_{n}^{-1} \mathbf{G}\right)^{-1} \mathbf{G}^{H} \mathbf{C}_{n}^{-1} \mathbf{y}
$$

In (9) $\mathbf{C}_{n}$ is the covariance matrix for the interference plus noise terms in (8). The conventional MMSE receiver assumes that interference is uncorrelated across the receiving antennas.

In this case $\mathbf{C}_{n}$ would be given by $\mathbf{C}_{n}=\left(\gamma^{-1}+f^{-1}\right) \mathbf{I}_{n_{r}}$. The MMSE-IRC takes the interference correlation into consideration. In this case $\mathbf{C}_{n}=\mathbf{C}_{i}+\mathbf{C}_{v}$, where $\mathbf{C}_{i}$ is the interference covariance matrix, which needs to be estimated. Methods for estimating $\mathbf{C}_{i}$ are available in the literature and are outside the scope of this paper. For example the 3GPP document [21, section 4.3] provides an algorithm for this estimation using the LTE reference signals. In this paper we assume perfect knowledge of the interference covariance matrix. Hence, $\mathbf{C}_{n}$ is given from (8) by: 


$$
\mathbf{C}_{n}=\left(\gamma^{-1} \mathbf{I}_{n_{r}}+f^{-1} \mathbf{G}_{i} \mathbf{G}_{i}^{H}\right)
$$

Hence, the MMSE-IRC receiver is given by:

$$
\hat{\mathbf{s}}=\left(\mathbf{I}_{n_{l}}+\mathbf{G}^{H} \mathbf{C}_{n}^{-1} \mathbf{G}\right)^{-1} \mathbf{G}^{H} \mathbf{C}_{n}^{-1} \mathbf{y}
$$

\section{SIMULATION RESULTS}

A CoMP network with a UE at equal distance from $3 \mathrm{eNBs}$ is simulated. The employed channel model is the Extended Typical Urban (ETU) and Extended Pedestrian (EPA) specified by LTE [28, Annex B]. The delay spread of the ETU channel model is higher than the EPA model. Since antenna correlation has a significant effect on the performance of CoMP and MMSE-IRC we consider low and medium correlation [29, Annex B]. We compare eNB selection as in section III above to the case of UE receiving from the first 2 eNBs (denoted as no eNB selection). We also compare local and global precoding matrix selection. In addition, we compare the performance of MMSE and MMSE-IRC as in section V. we study the effect of the desired to interference eNB power ratio $f$ in case of medium correlation for 3 and 4 layers using MMSE-IRC receiver. Also the BER performance between 3 and 4 layers in case of different values of the desired to interference eNB power ratio $f$ is compared.

Table I shows the simulation parameters. In this paper, we ignore the feedback latency of the precoding matrix selection (i.e., sufficiently slow fading channel).

Note that the UE is allocated 5 Resource Blocks $(900 \mathrm{kHz})$. This moderate bandwidth justifies fixing the precoding matrix over all subcarriers.

All results show the coded BER versus $E_{b} / N_{o}$, defined by $E_{b} / N_{o}=\gamma /(Q R)$, where $\gamma$ is the SNR defined before, $R$ is the code rate and $Q$ is the modulation index.

TABLE I. SIMITULATION PARAMETER

\begin{tabular}{|l|l|}
\hline \multicolumn{1}{|c|}{ Parameter } & \multicolumn{1}{c|}{ Value } \\
\hline Number of eNBs & 2 desired + 1 interference \\
\hline TTI size & 14 OFDM symbols (normal CP LTE) \\
\hline System bandwidth & $20 \mathrm{MHZ}$ \\
\hline Channels coding scheme & Turbo coding, Log Map, rate $R=1 / 3$ \\
\hline Type of receiver & MMSE, MMSE-IRC \\
\hline Modulation scheme & QPSK $(Q=2)$ \\
\hline Number of Resource Blocks for UE & 5 \\
\hline Fading channel model & $\begin{array}{l}\text { ETU, EPA with prefect channel } \\
\text { estimation }\end{array}$ \\
\hline Detection algorithm & MMSE, MMSE-IRC \\
\hline
\end{tabular}


International Journal of Wireless \& Mobile Networks (IJWMN) Vol. 7, No. 3, June 2015

\begin{tabular}{|l|l|}
\hline Power ratio desired/interference per eNB & $f=0,3,10 \mathrm{~dB}$ \\
\hline $\begin{array}{l}\text { Number of transmit antennas = number of receive } \\
\text { antenna }\end{array}$ & $n_{t}=n_{r}=4$ \\
\hline Number of layers & $n_{l}=3$ or 4 \\
\hline Wireless frame Lengths & $10 \mathrm{~ms}$ \\
\hline Sub-frame length & $1 \mathrm{~ms}$ \\
\hline Length of time slot & $0.5 \mathrm{~ms}$ \\
\hline Sampling frequency & $32.72 \mathrm{MHz}$ \\
\hline Sub-carrier interval & $15 \mathrm{KHz}$ \\
\hline
\end{tabular}

Figure 6 compares local and global precoding matrix in the cases with and without selecting the 2 jointly transmitting eNBs and 1 interference eNB. For this figure we employ low antenna correlation with ETU channel, $f=3 \mathrm{~dB}$ and $n_{l}=3$ layers using MMSE-IRC receiver. It is clear from this figure that global precoding matrix selection considerably outperforms local precoding matrix selection. Also, for both cases, selecting the 2 jointly transmitting eNB provides additional performance gain. This justifies the extra processing in the UE side.

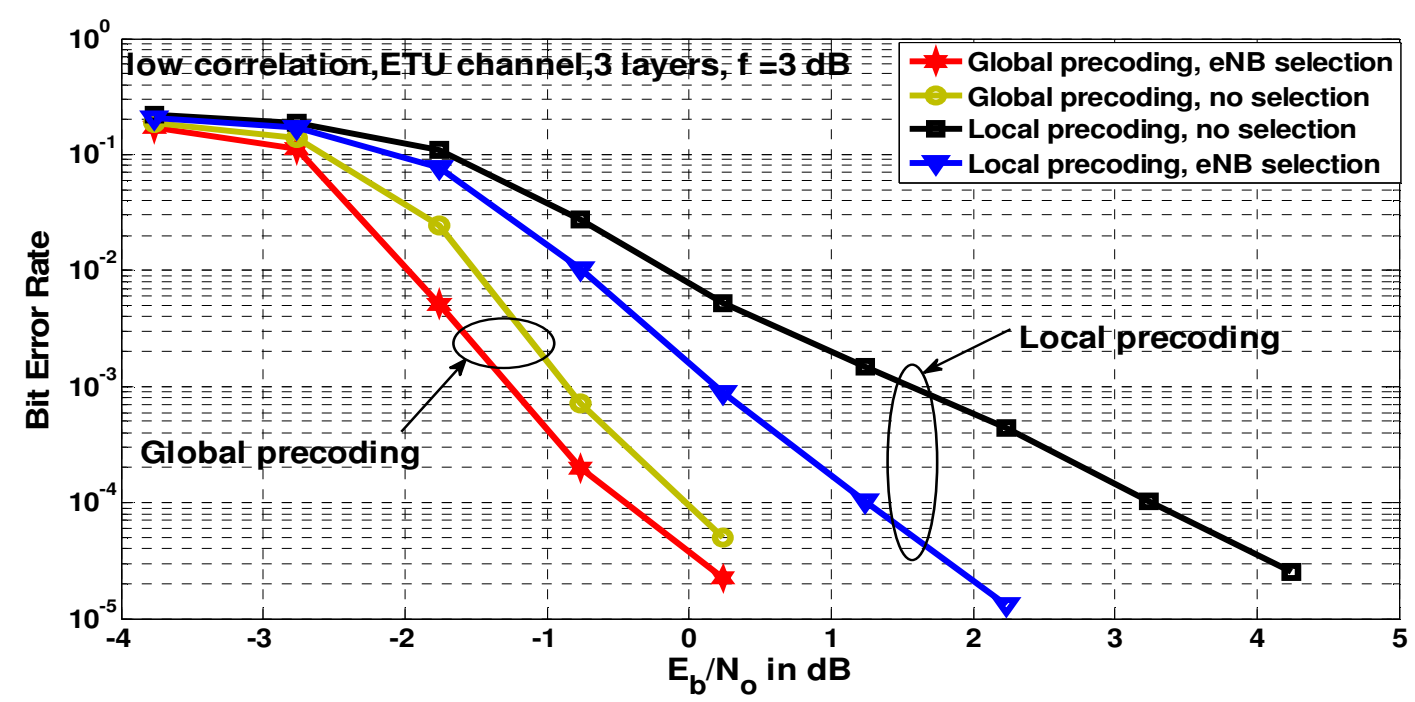

Figure 6. BER of global and local precoding matrix in CoMP-JT with and without cell selection at low correlation using IRC receiver for 3 layers and $f=3 \mathrm{~dB}$ under ETU channel model.

Figure 7 repeats that same results of Figure 6 but for EPA channel. Compared to Figure 6, The ETU channel shows a better performance than the EPA due to the increased frequency selectivity. 


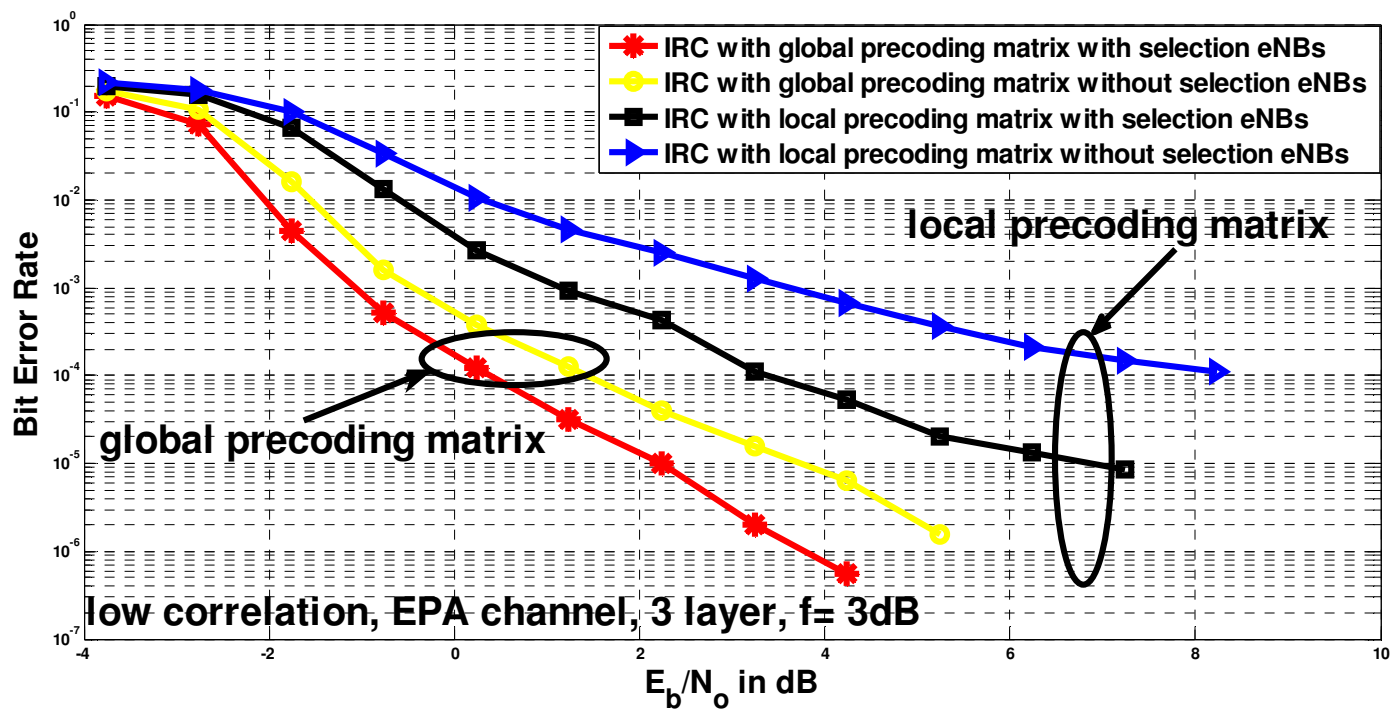

Figure 7. BER of global and local precoding matrix in CoMP-JT with and without cell selection at low correlation using IRC receiver for 3 layers and $f=3 \mathrm{~dB}$ under EPA channel model.

Figure 8 repeats that same results of Figure 6 but for $n_{l}=4$ layers. Compared to Figure 6, not surprisingly the 3 layers case shows lower BER than the 4 layers case. It is interesting to see that the relative gain that is achieved due to selecting the jointly transmitting eNBs and global precoding matrix selection is higher than the case of 3 layers. This supports our claim that the additional processing and overhead needed to implement the techniques presented in this paper are well paid-back for in terms of improved performance.

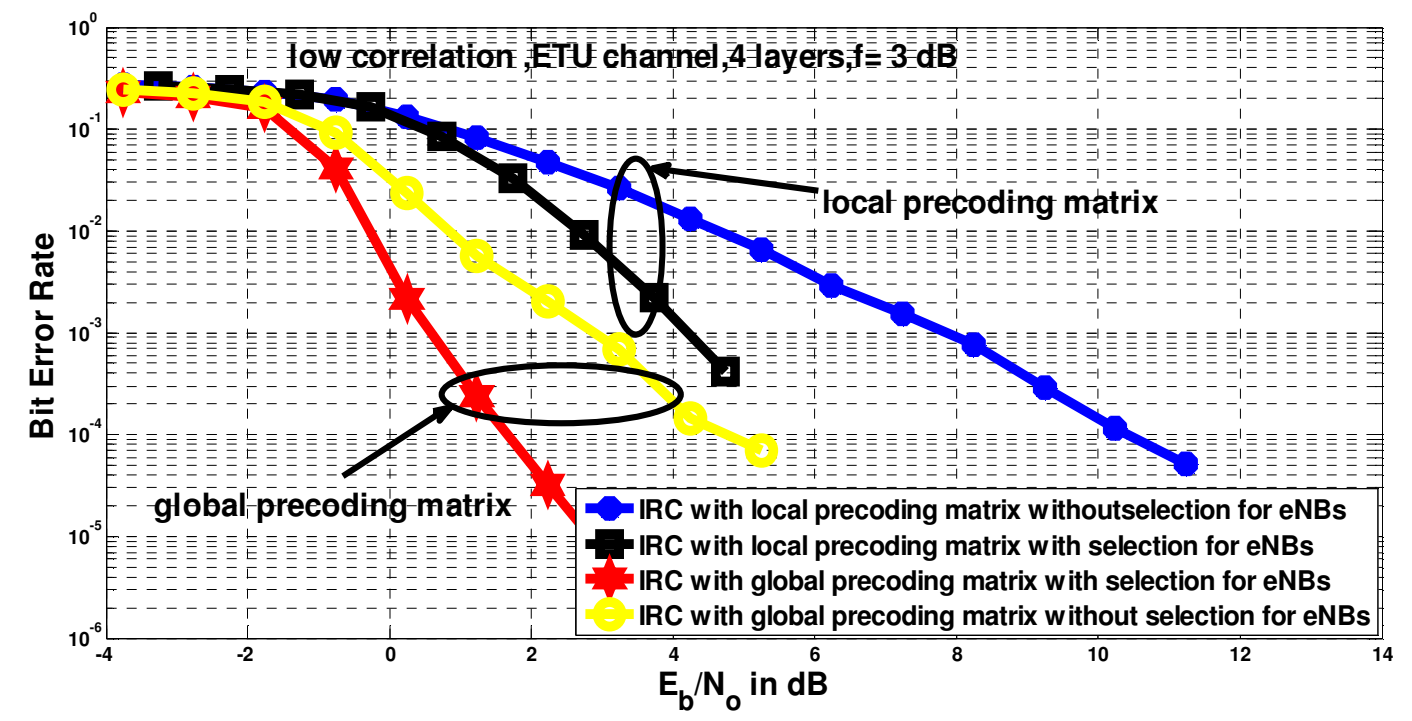

Figure 8. BER of global and local precoding matrix in CoMP-JT with and without cell selection at low correlation using IRC receiver for 4 layers and $f=3 \mathrm{~dB}$ under ETU channel model.

Figure 9 repeats that same results of Figure 6 but for MMSE receiver. Compared to Figure 6, it is clear from these figures that the MMSE-IRC improves the performance considerably compared to the MMSE. 


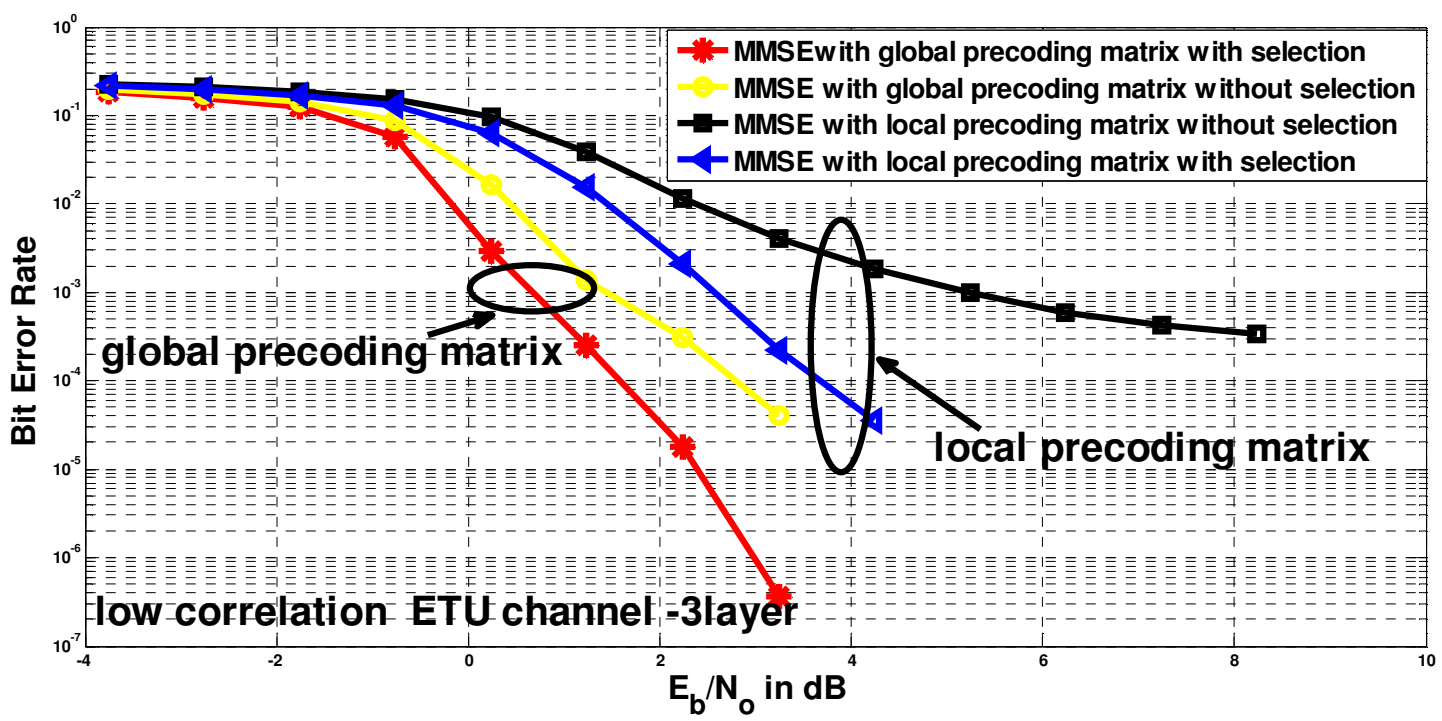

Figure 9. BER of global and local precoding matrix in CoMP-JT with and without cell selection at low correlation using MMSE receiver for 3 layers and $f=3 \mathrm{~dB}$ under ETU channel model.

Figure 10 repeats that same results of Figure 8 but for MMSE receiver. Compared to Figure 8, it is clear from these figures that the MMSE-IRC improves the performance considerably compared to the MMSE.

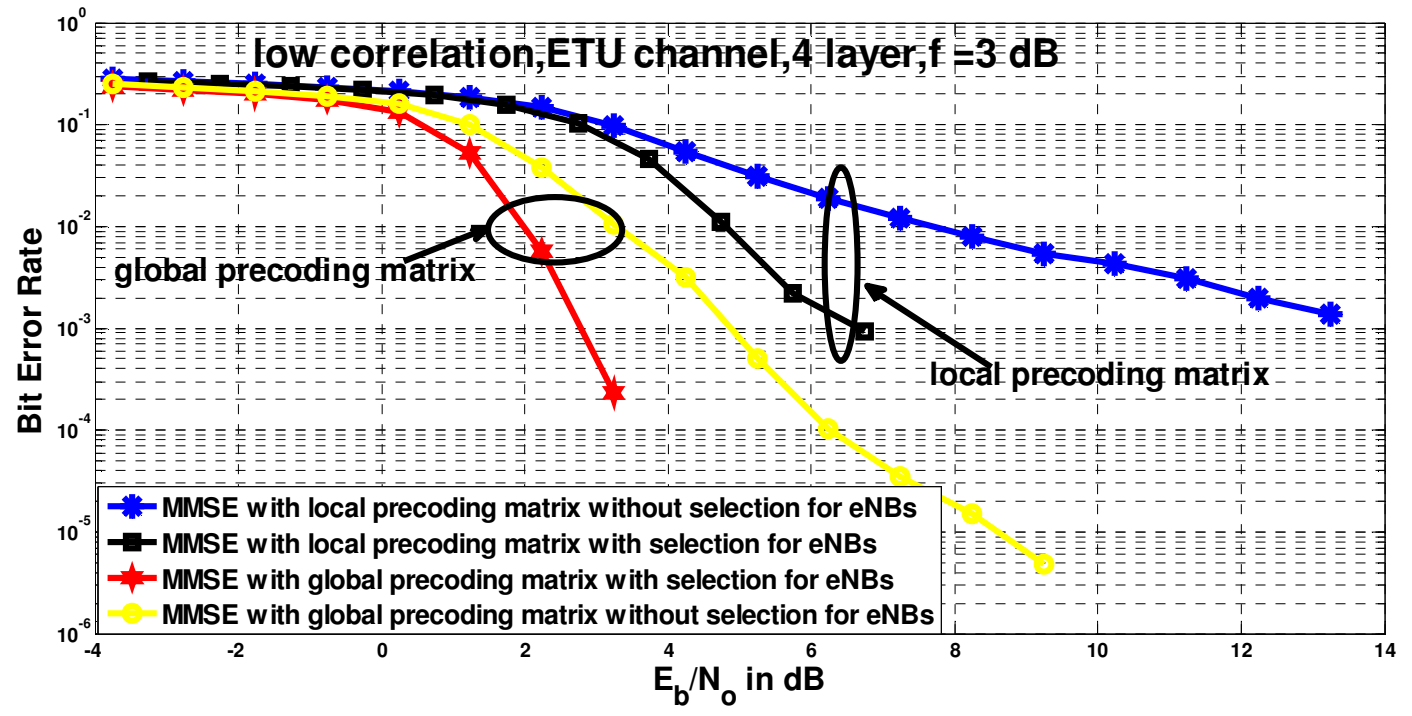

Figure 10. BER of global and local precoding matrix in CoMP-JT with and without cell selection at low correlation using MMSE receiver for 4 layers and $f=3 \mathrm{~dB}$ under ETU channel model.

Figure 11 repeats that same results of Figure 7 but for MMSE receiver. Compared to Figure 7, it is clear from these figures that the MMSE-IRC improves the performance considerably compared to the MMSE. 
International Journal of Wireless \& Mobile Networks (IJWMN) Vol. 7, No. 3, June 2015

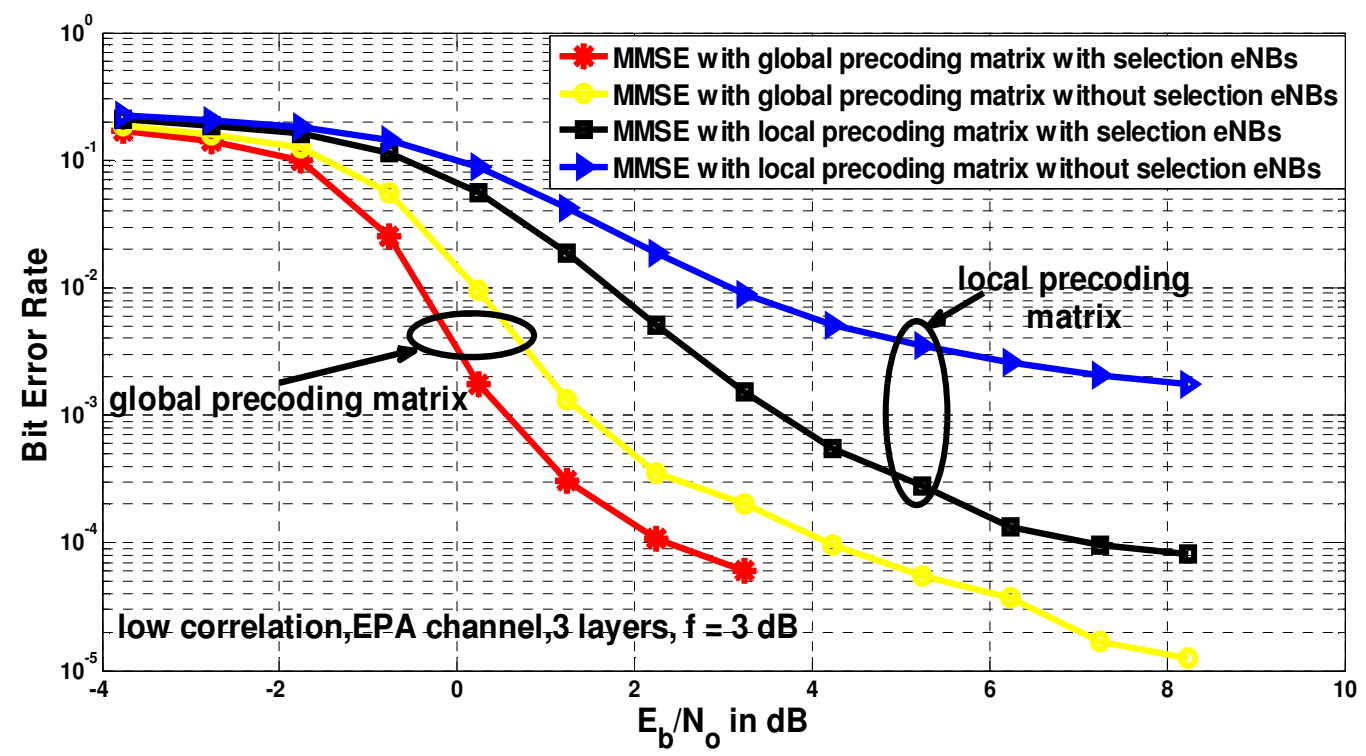

Figures 11. BER of global and local precoding matrix in CoMP-JT with and without cell selection at low correlation using MMSE receiver for 3 layers and $f=3 \mathrm{~dB}$ under EPA channel model.

Figures 12 and 13 compares MMSE and MMSE-IRC in case global and local precoding matrix respectively and with selecting the 2 jointly transmitting eNBs and 1 interference eNB. For this figures we employ low antenna correlation with $f=0 \mathrm{~dB}$ and $n_{l}=4$ layers under ETU channel model. It clear from figures that the MMSE-IRC improves the performance considerably compared to the MMSE.

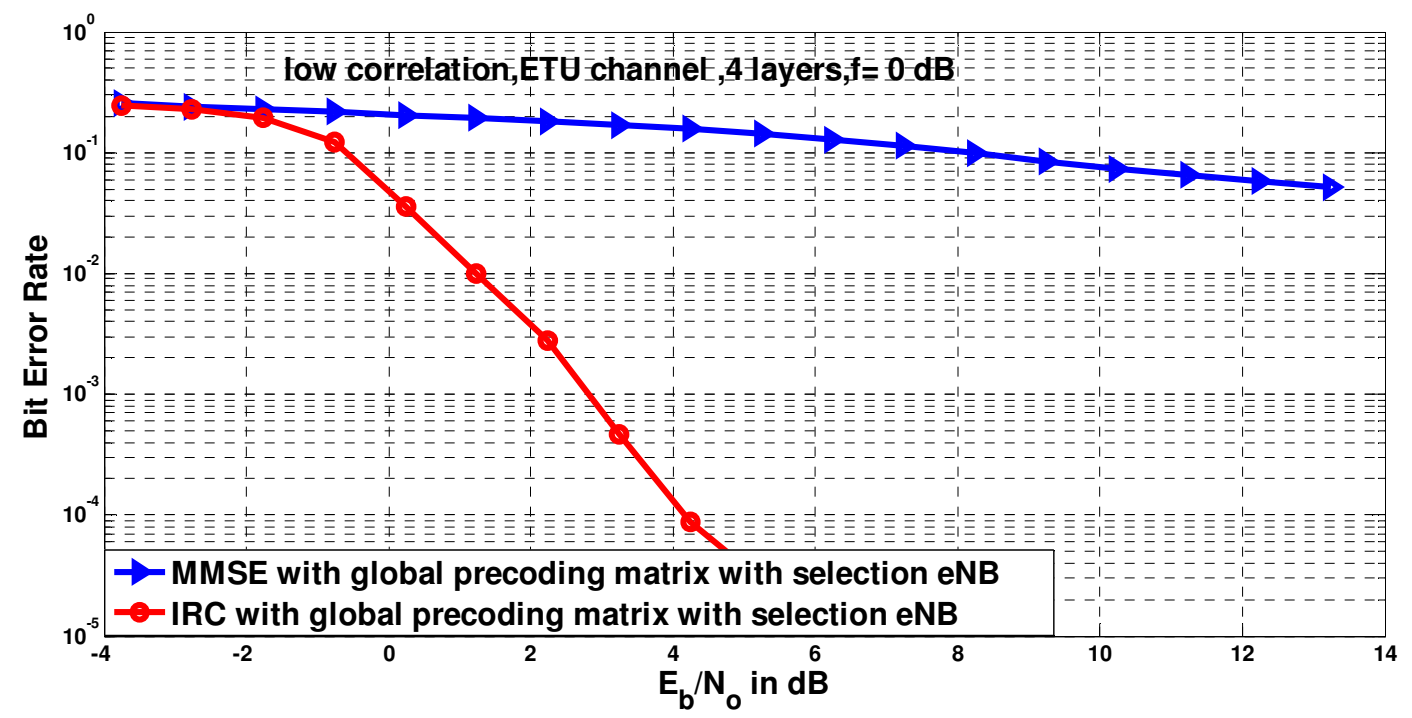

Figure 12. Comparison between MMSE and MMSE-IRC with global precoding matrix and with cell selection at low correlation and $f=0 \mathrm{~dB}$ under ETU channel model. 


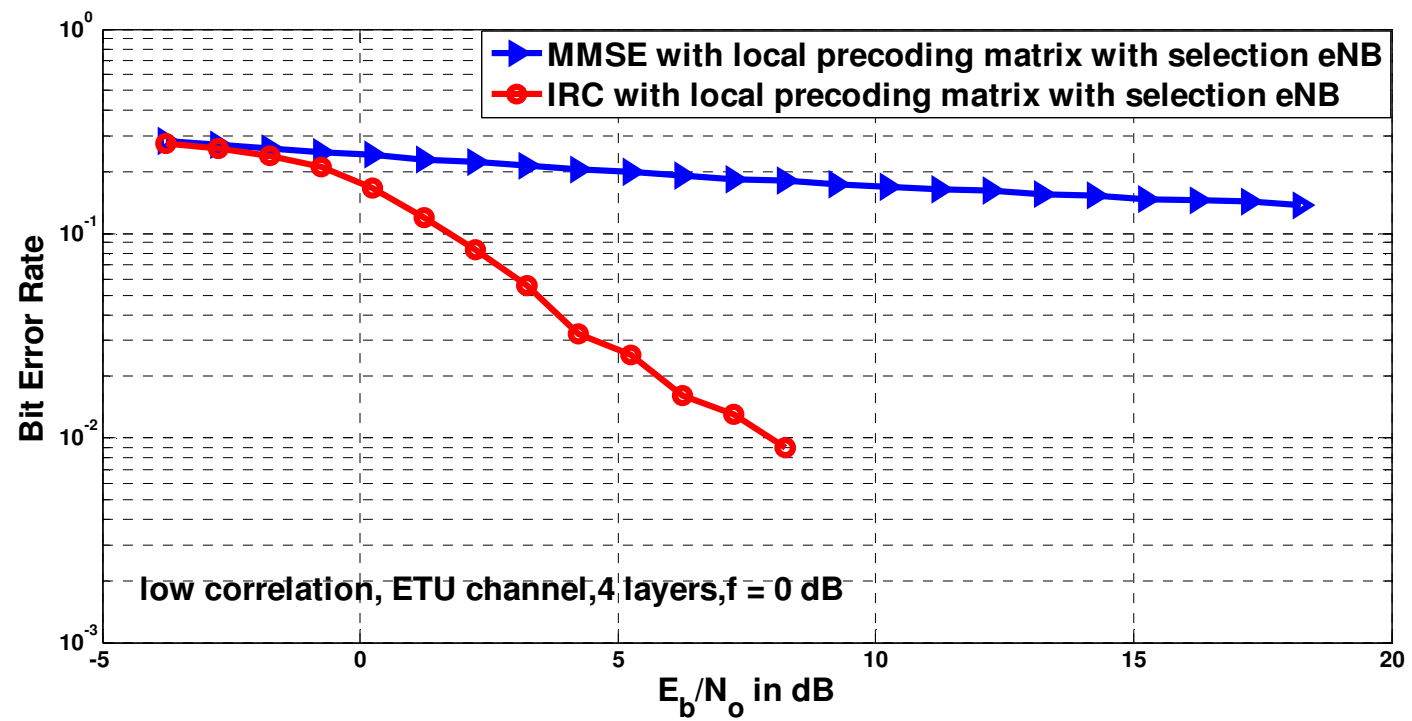

Figure 13. Comparison between MMSE and MMSE-IRC with local precoding matrix and with cell selection at low correlation and $f=0 \mathrm{~dB}$ under ETU channel model.

Figures 14 and 15 repeat that same results of Figure 6 but for medium antenna correlation under ETU and EPA channel model respectively. Compared to Figure 6, we can observe an overall degradation in performance, which is expected to due to the loss in diversity. However, the relative gain that is achieved due to selecting the jointly transmitting eNBs and global precoding matrix selection is higher that the case of low correlation. This indicates that the methods described in this paper are more rewarding in the presence of antenna correlation. By comparing Figures 14 and 15, we found that the ETU channel shows a better performance than the EPA due to the increased frequency selectivity.

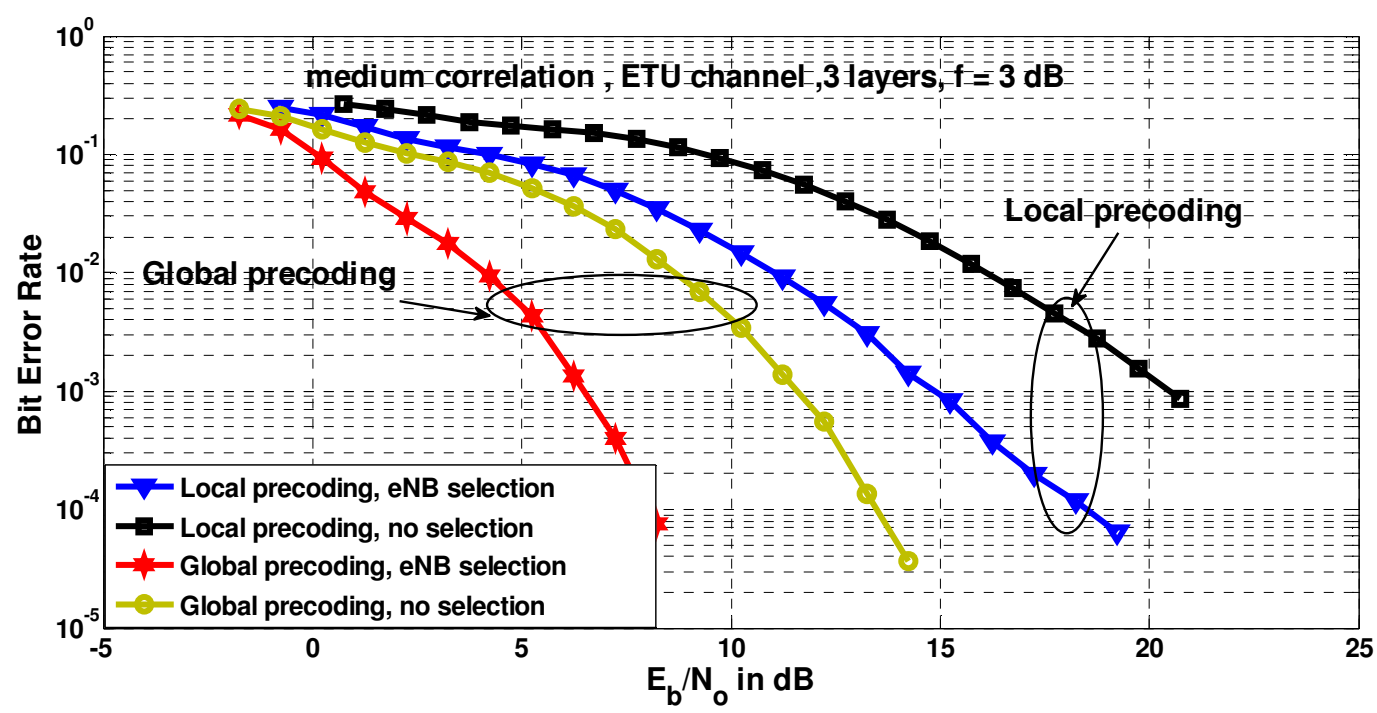

Figure 14. BER of global and local precoding matrix in CoMP-JT with and without cell selection at medium correlation using IRC receiver for 3 layers and $f=3 \mathrm{~dB}$ under ETU channel model. 


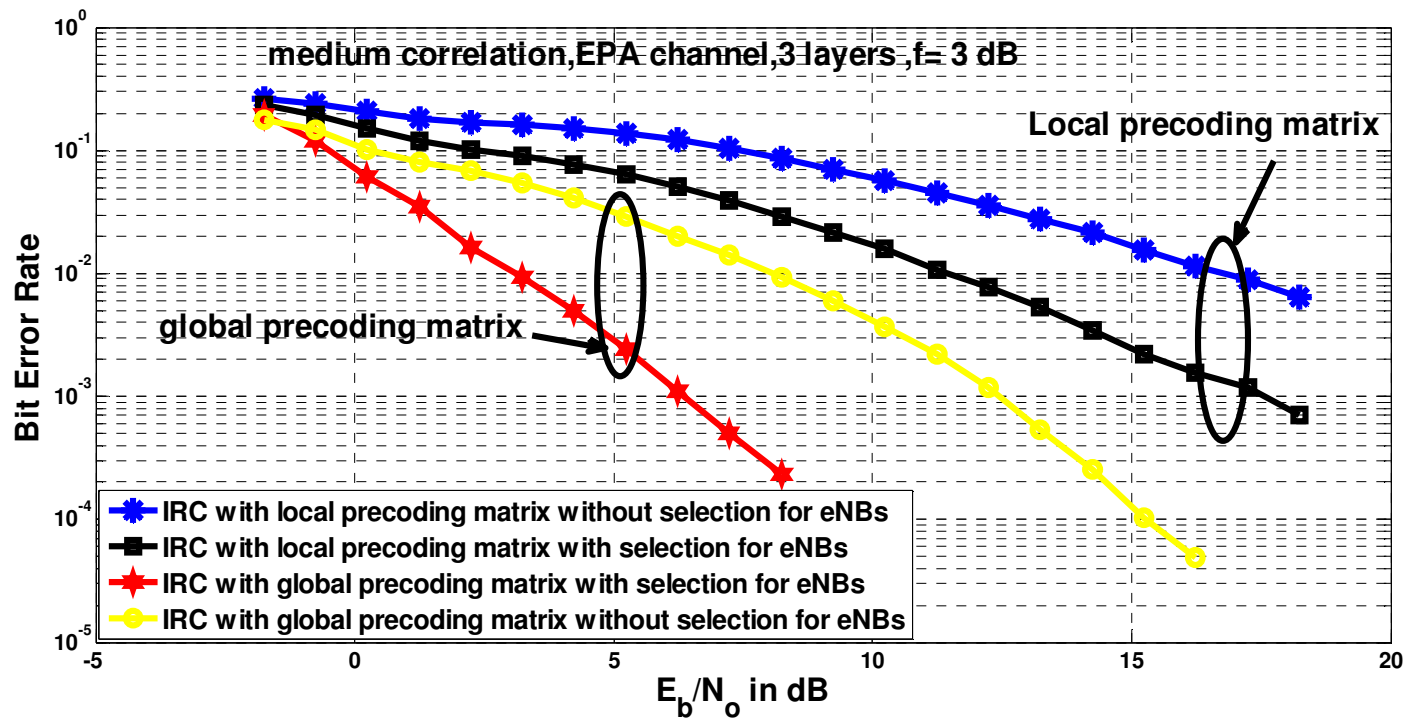

Figure 15. BER of global and local precoding matrix in CoMP-JT with and without cell selection at medium correlation using IRC receiver for 3 layers and $f=3 \mathrm{~dB}$ under EPA channel model.

Figure 16 repeats that same results of Figure 8 but for medium antenna correlation. Compared to Figure 8 we can observe an overall degradation in performance, which is expected to due to the loss in diversity. However, the relative gain that is achieved due to selecting the jointly transmitting eNBs and global precoding matrix selection is higher that the case of low correlation. This indicates that the methods described in this paper are more rewarding in the presence of antenna correlation.

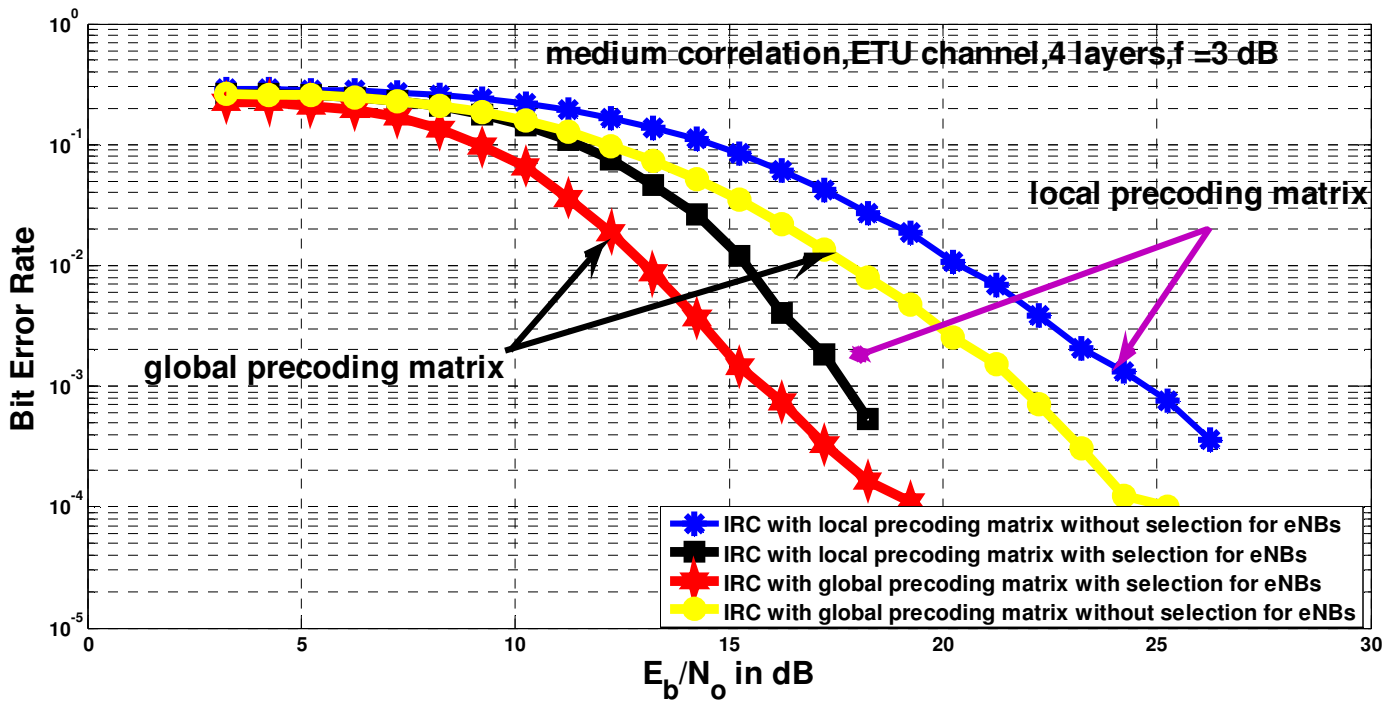

Figure 16. BER of global and local precoding matrix in CoMP-JT with and without cell selection at medium correlation using IRC receiver for 4 layers and $f=3 \mathrm{~dB}$ under ETU channel model.

Figures 17, 18 and 19 compare the BER performance of $n_{l}=3$ and 4 layers in case of $f=10,3$ and $0 \mathrm{~dB}$ respectively. To avoid crowding the figure we show only the two extreme cases: local 
precoding without CoMP eNBs selection (worst performance) and global precoding with CoMP eNBs selection (best performance). Not surprisingly the 3 layers case shows lower BER than the 4 layers case (at the cost of lower bit rate). However, it is interesting to see that the difference between the two 4-layers curves is much larger than the difference between the two 3-layers curves. This indicates that the gain realized by the techniques presented in this paper is much higher in 4 layers case with higher bit rate. Also, we can observe an overall degradation in performance in case of $f=0 \mathrm{~dB}$, which is expected. However, the relative gain that is achieved due to selecting the jointly transmitting eNBs and global precoding matrix selection is higher than the case of $f=10$ and $3 \mathrm{~dB}$. This supports our claim that the additional processing and overhead needed to implement the techniques presented in this paper are well paid-back for in terms of improved performance.

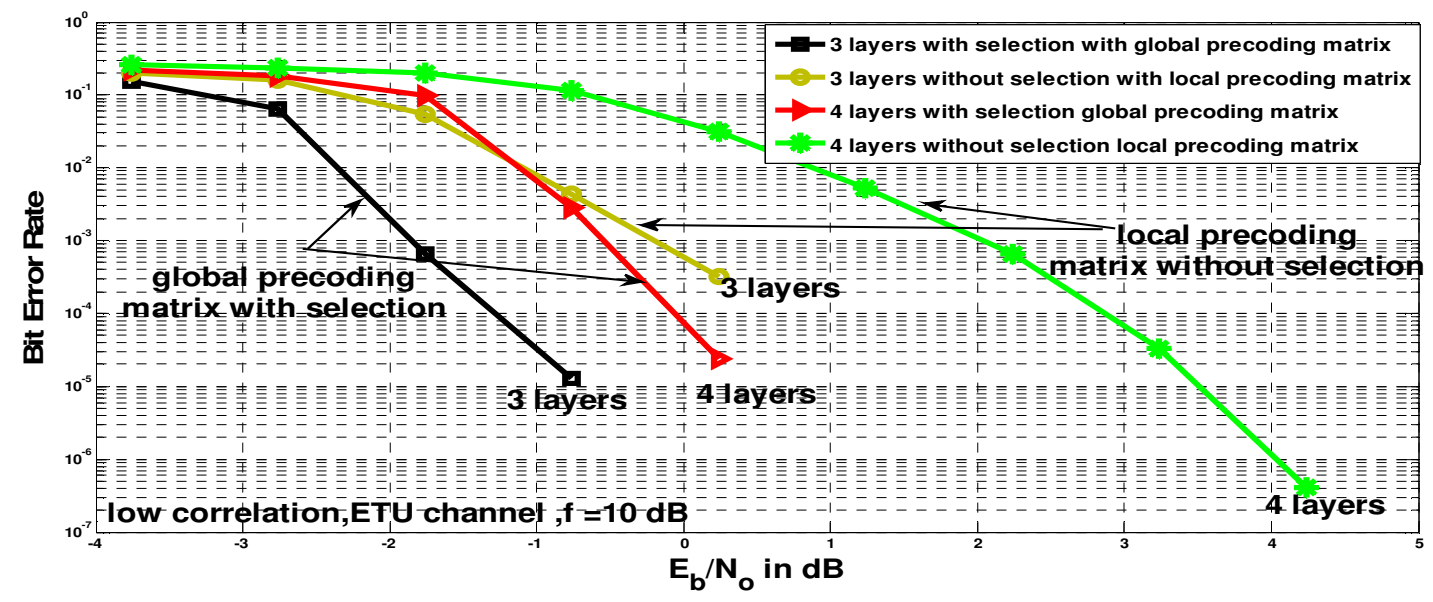

Figure 17. BER comparison between 3 and 4 layers at low correlation $f=10 \mathrm{~dB}$ under ETU channel model.

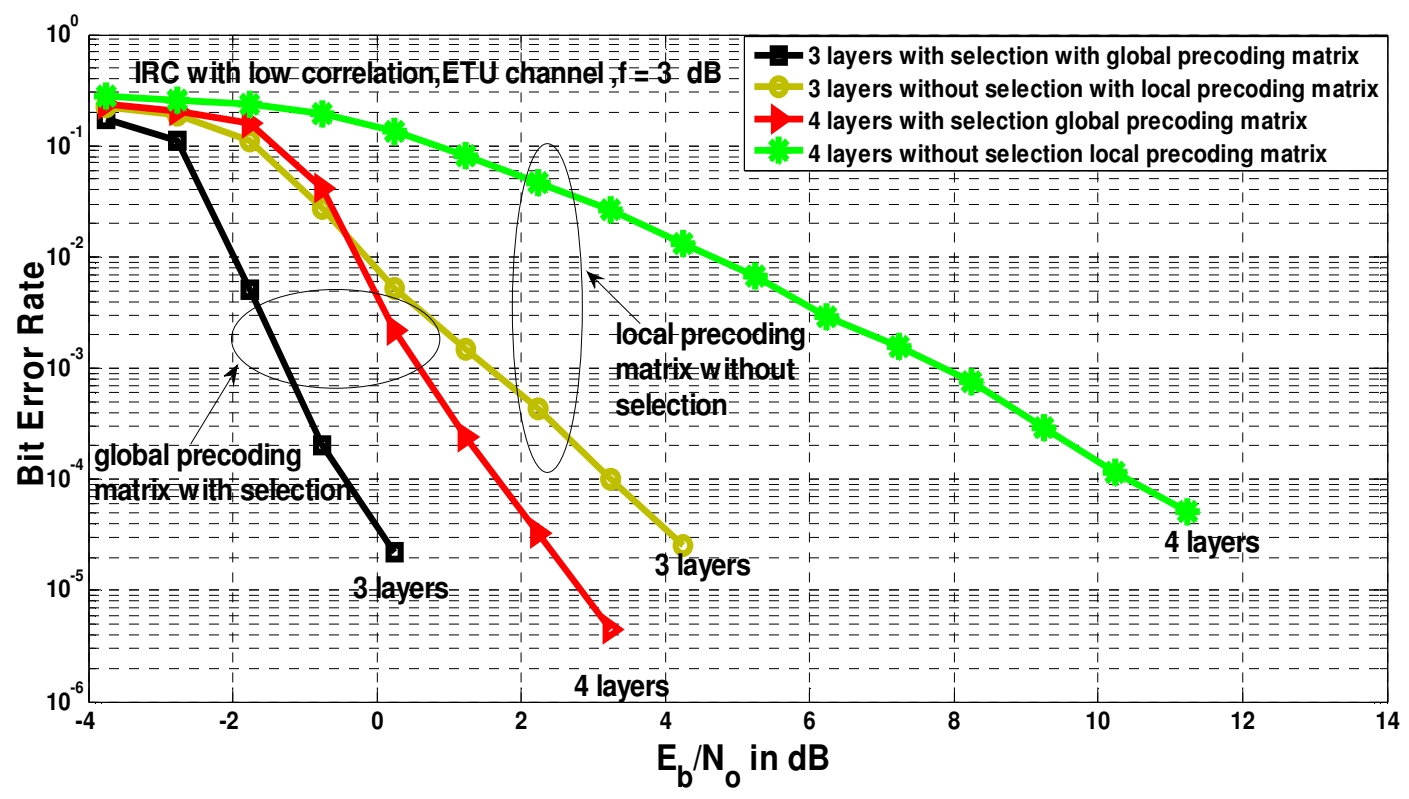

Figure 18. BER comparison between 3 and 4 layers at low correlation $f=3 \mathrm{~dB}$ under ETU channel model. 


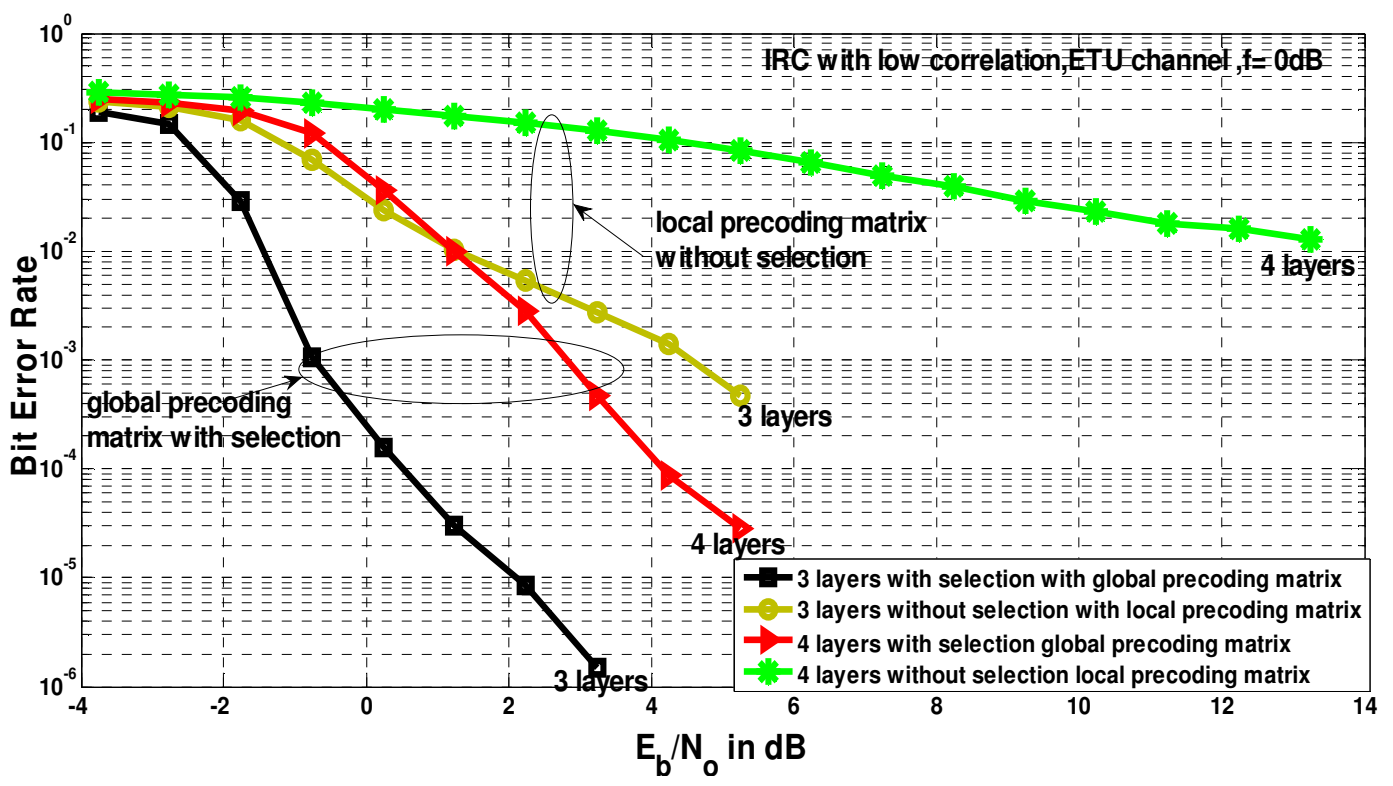

Figure 19. BER comparison between 3 and 4 layers at low correlation $f=0 \mathrm{~dB}$ under ETU channel model.

Figures 20,21, 22 and 23 study the effect of the desired to interference eNB power ratio $f$ in case of medium correlation for 3 and 4 layers respectively using MMSE-IRC receiver. For Figures 20 and 21 we employ global precoding matrix while Figures 22 and 23 we employ local precoding matrix. It is clear from those figures that increasing value of $f$ (interference power reduction) gives better performance. This supports the need for power control in LTE.

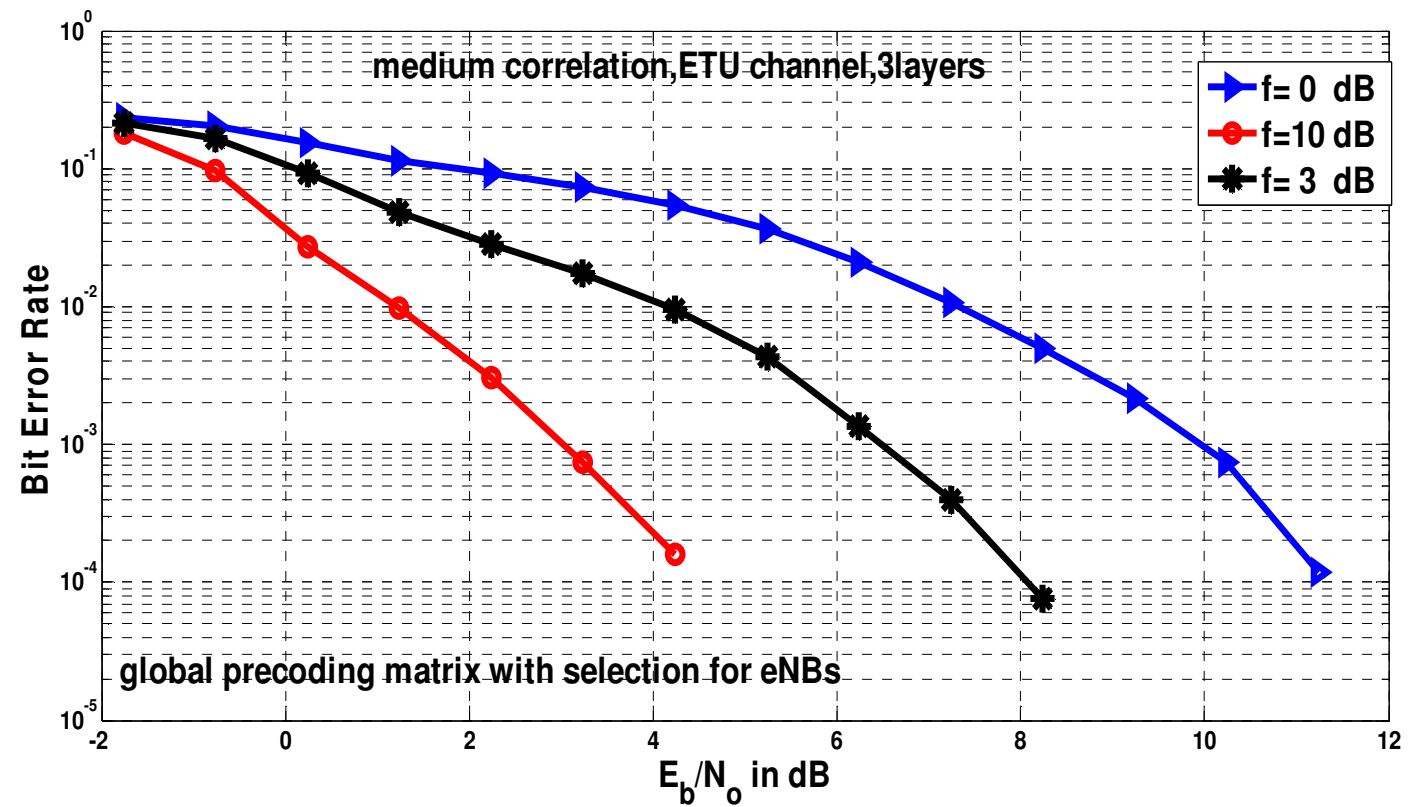

Figure 20. BER comparison between different values for $f$ in case of 3 layers at medium correlation using MMSE-IRC receiver under ETU channel for global precoding matrix with selection. 


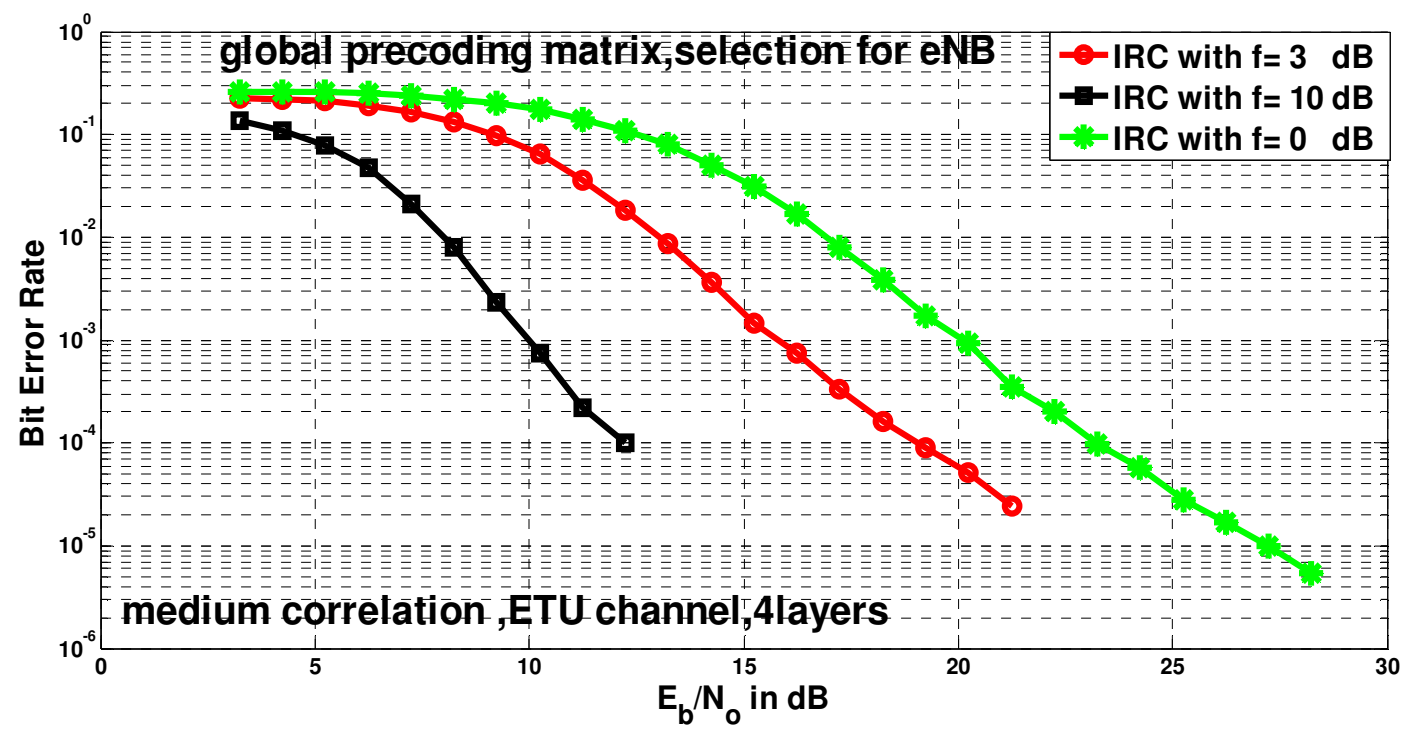

Figure 21. BER comparison between different values for $f$ in case of 4 layers at medium correlation using MMSE-IRC receiver under ETU channel for global precoding matrix with selection.

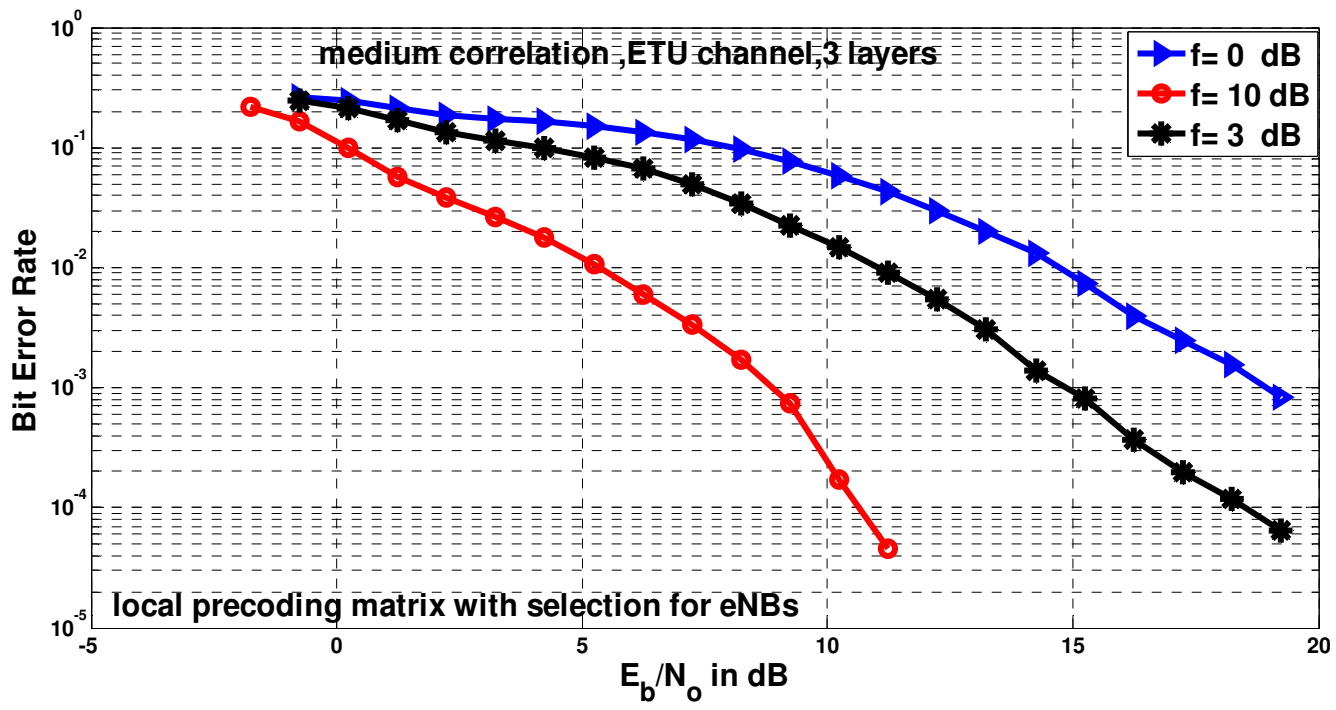

Figure 22. BER comparison between different values for $f$ in case of 3 layers at medium correlation using MMSE-IRC receiver under ETU channel for local precoding matrix with selection. 


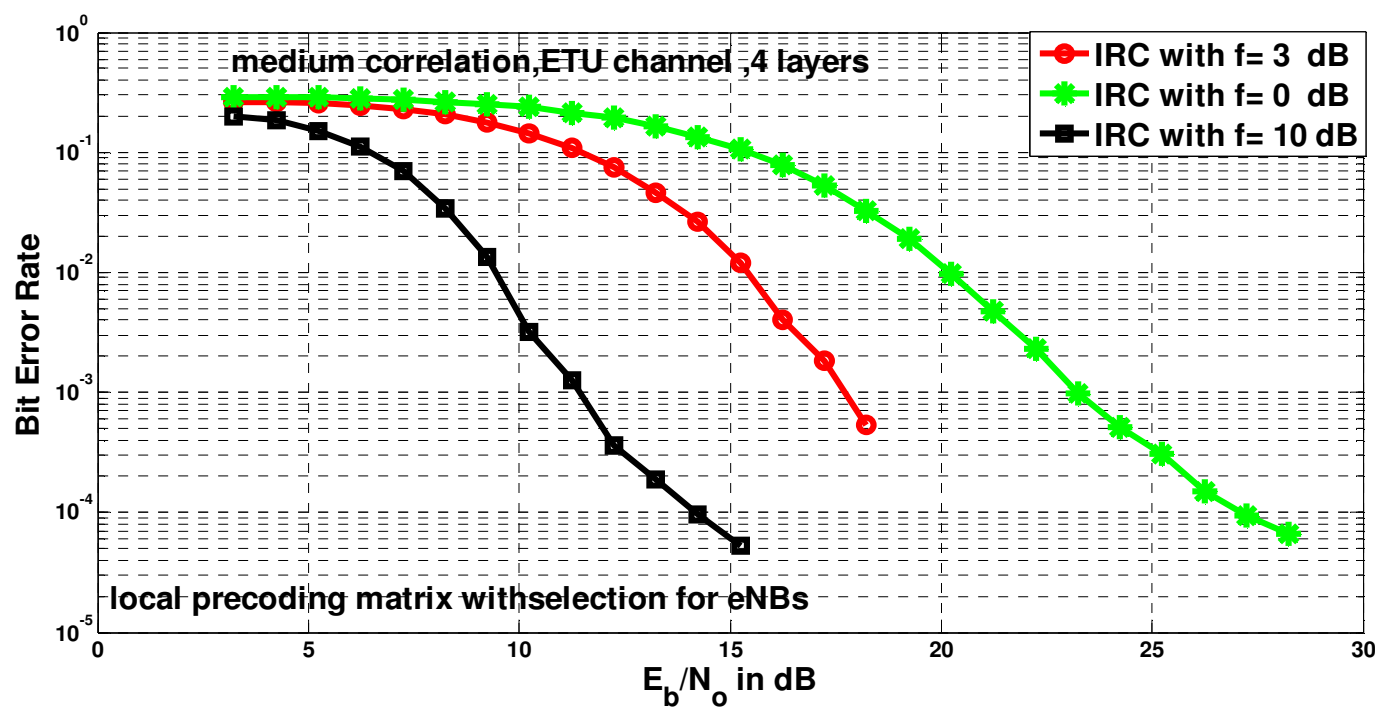

Figure 23. BER comparison between different values for $f$ in case of 4 layers at medium correlation using MMSE-IRC receiver under ETU channel for local precoding matrix with selection.

\section{CONCLUSION}

In this paper, the LTE-A downlink performance is presented in the case of CoMP-JT with local and global precoding matrix selection with and without dynamic transmitting eNB selection. We conclude that global precoding matrix selection outperforms the conventional local precoding matrix and dynamic selection for CoMP eNBs gives better performance in all cases. The relative gain that is achieved due to selecting the jointly transmitting eNBs and global precoding matrix selection in case of medium correlation is higher that the case of low correlation. This indicates that the methods described in this paper are more rewarding in the presence of antenna correlation. This applies to MMSE and MMSE-IRC. In all cases MMSE-IRC gives much better performance than MMSE receiver. Also we conclude that increasing value of the power ratio between the desired signal from each desired eNB and the interference signal gives better performance. This supports the need for power control in LTE. We found that the ETU channel shows a better performance than the EPA due to the increased frequency selectivity.

\section{REFERENCES}

[1] P. Bhat, S. Nagata, L. Campoy, I. Berberana, T. Derham, G. Liu, X. Shen, P. Zong and J. Yang, Verizon, "LTE-Advanced: An Operator Perspective," IEEE Communications Magazine, February 2012.

[2] ITU-R Rec. M.1645, "Framework and Overall Objectives of the Future Development of IMT-2000 and Systems Beyond IMT-2000," 2003.

[3] S. Parkvall and D. Astely, "The Evolution of LTE Toward LTE Advanced," J. Commun., vol. 4, pp. 146-54, Apr. 2009.

[4] “ITU Paves Way for Next-Generation 4G Mobile Technologies,"ITU press release, 21 Oct. 2010.

[5] S. Sesia, I. Toufik and M. Baker "LTE - The UMTS Long Term Evolution: From Theory to Practice,” John Wiley \& Sons, Ltd., 2009, ISBN: 978-0-470-69716-0.

[6] D. Gesbert, M. Shafi, Da-shan Shiu, P.J. Smith and A. Naguib, "From Theory to Practice: An Overview of MIMO Space-Time Coded Wireless Systems," IEEE Journal on Selected Areas in Communications, vol. 21, no.3, pp. 281-302, April 2003.

[7] D. Gesbert, M. Kountouris, R. Heath, C. -B. Chae and T. Salzer, "Shifting the MIMO Paradigm," IEEE Singal Processing Magazine, vol. 24, no. 5, pp. 36-46, September 2. 
International Journal of Wireless \& Mobile Networks (IJWMN) Vol. 7, No. 3, June 2015

[8] L. Liu, R. Chen, K. Sayana, Z. Shi ,Y. Zhou and S. Geirhofer "Downlink MIMO in LTE- Advanced: SU-MIMO vs. MU-MIMO,” IEEE Communications Magazine , February 2012 0163-6804.

[9] J. Lee, J. Han, and J. Zhang, "MIMO technologies in 3GPP LTE and LTE-Advanced," EURASIP Journal on Wireless Communications and Networking, Vol 2009, May 20.

[10] M. Sawahashi, Y. Kishiyama, D. Nishikawa and M. Tanno "Coordinated Multipoint Transmission/Reception Techniques for Lte-Advanced,” IEEE Wireless Communications, June 2010 1536-1284.

[11] H. Dung, C. Zhu, Y. Xu, Y. Wang, Z. Ding "Joint Transmission Using Global Code word and Codebook Design for Coordinated Multipoint Processing (CoMP)," Globecom Workshops (GC Wkshps), Anaheim, CA, Dec. 2012, pp. 1118 - 1122.

[12] H. Määttänen, K. Hämäläinen , J. Venäläinen , K. Schober, M. Enescu and M. Valkama, "Systemlevel performance of LTE-Advanced with joint transmission and dynamic point selection schemes," EURASIP Journal on Advances in Signal Processing 2012, 2012:247. http://asp.eurasipjournals.com/content/2012/1/247.

[13] C. Yang, S. Han, X. Hou and A. Molisch, "How do we design CoMP to achieve its promised potential?" IEEE Wireless Communications, February 2013.

[14] J. Lee, Y. Kim, and H. Lee, B. Loong, D.Mazzarese, J.Liu, W. Xiao, and Y. Zhou, "Coordinated Multipoint Transmission and Reception in LTE-Advanced Systems," IEEE Communications Magazine, November 2012.

[15] H. Raafat, E. Sourour and H. Elkamchouchi "Performance of Joint Transmission CoMP With Global Precoding Matrix and IRC Receiver for LTE-A,” International Conference on New Technologies, Mobility and Security (NTMS), vol.7, Paris, France, July 2015.

[16] 3GPP TR 36.211 v11.4.0, "Evolved Universal Terrestrial Radio Access (E-UTRA); Physical channels and modulation Release 11," September. 2013.

[17] A. Davydov, G. Morozov and A. Papathanassiou "Advanced Interference Suppression Receiver for LTE-Advanced Systems," International Symposium on Personal, Indoor and Mobile Radio Communications' 2013, vol.24, 978-1-4577-1348-4.

[18] 3GPP TR 36.829, "Enhanced performance requirement for LTE User Equipment (UE)," V.11.1.0, December 2012.

[19] Y. Ohwatari, N. Miki, Y. Sagae, Y. Okumura "Investigation on Interference Rejection Combining Receiver for Space-Frequency Block Code Transmit Diversity in LTE-Advanced Downlink," IEEE Trans. on Vehicular Technology, January 2014, Vol. 63, pp 191 - 203.

[20] H. Kai, J. Fujiang "An Improved Signal Detection Algorithm of LTE System in Interference Environment," Journal of Networks, March 2014, Vol. 9, pp793-798.

[21] Y. Sagae, Y. Ohwatari and Y. Sano "Improved Interference Rejection and Suppression Technology in LTE Release 11 Specifications," NTT DOCOMO Technical Journal, 2013 Vol. 15.

[22] 3GPP TR 36.829 V.11.1.0, "Enhanced performance requirement for LTE User Equipment (UE), December 2012.

[23] K. Kwak, H. Lee, H. Je, J. Hong and S. Choi, "Adaptive and Distributed CoMP Scheduling in LTEAdvanced Systems," IEEE 78th Vehicular Technology Conference (VTC Fall), 2013, pp.1 to 5, 2-5 Sept. 2013.

[24] R. Heath, S. Sandhu, and A. Paulraj, "Antenna selection for spatial multiplexing systems with linear receivers," IEEE Communications Letters, vol.5, no.4, pp.142, 144, April 2001.

[25] D. Love and R. Heath, "Limited feedback unitary precoding for spatial multiplexing systems," IEEE Transactions on Information Theory, vol. 51, no. 8, pp. 2967 to 2976, Aug. 2005.

[26] 3GPP TR 36.211 v11.4.0, "Evolved Universal Terrestrial Radio Access (E-UTRA); Physical channels and modulation Release 11," September. 2013.

[27] J. Wang "Decorrelation Based Receive Transformation for MIMO System under Multi-user CoChannel Interference," IEEE Wireless Communications Letters, VOL. 3, NO. 3, June 2014.

[28] 3GPP TR 36.521, "User Equipment (UE) conformance specification Radio transmission and reception," V.9.2, September 2010.

[29] 3GPP TR 36.101 v12.7.0, "Evolved Universal Terrestrial Radio Access (E-UTRA); User Equipment (UE) radio transmission and reception, Release 12," March. 2015. 